\title{
Restorative Plasticity of Dopamine Neuronal Transplants Depends on the Degree of Hemispheric Dominance
}

\author{
Guido Nikkhah, Gero Falkenstein, and Christoph Rosenthal \\ Neurosurgical Clinic, Nordstadt Hospital, D-30167 Hannover, Germany
}

The ability of dopaminergic (DA) transplants to restore complex sensorimotor behaviors in experimental Parkinson's disease is dependent on graft survival and reinnervation and is likely to be further modified by complex functional graft-host interactions. Here, we examined the impact of hemispheric dominance and extensive testing regimes on the functional capabilities of DA transplants to restore skilled forelimb movements in rats with unilateral 6-hydroxydopamine lesions. Interestingly, a near complete recovery was observed in DA-grafted animals that did not exhibit a strong hemispheric lateralization for paw use before lesion and implantation surgery, whereas animals with a clear lateralization of paw use and grafted into the contralateral hemisphere exhibited only moderate recovery. Finally, animals grafted ipsilateral to the preferred paw were most resistant to functional improvements in skilled forelimb use. However, the influence of hemispheric dominance on the degree of functional
DA graft-induced restoration was specific for skilled forelimb use, whereas no such differences were observed in other tests for motor and sensory functions related to the DA system. Furthermore, functional recovery of DA-grafted animals in skilled forelimb use was significantly promoted by extensive behavioral testing regimes indicative of a "learning how to use" the transplant effect.

These findings indicate the importance of the underlying functional architecture of complex sensorimotor behaviors, such as skilled forelimb use, and the DA neurotransmitter system for the plasticity of DA transplants to promoting a more complete behavioral recovery in experimental, and potentially, also in clinical forms of Parkinson's disease.

Key words: Parkinson's disease; 6-hydroxydopamine; skilled forelimb use; stepping; sensorimotor behavior; nigrostriatal dopamine system; hemispheric lateralization
Transplantation of embryonic dopaminergic (DA) grafts has developed from a neurobiological model into clinical applications in Parkinson's disease (PD) (Dunnett and Björklund, 1999). Unilateral injections of 6-hydroxydopamine (6-OHDA) into the medial forebrain bundle (MFB) result in a complete destruction of the mesostriatal dopamine system, which is widely considered as a good animal model for the anatomical and functional changes observed in human parkinsonism (Herman and Abrous, 1994). Previous studies have demonstrated that DA grafts survive, functionally integrate into the host neuronal circuitry, and thereby promote the behavioral recovery of a variety of sensorimotor deficits induced by the degeneration of the intrinsic dopamine system (Björklund, 1992; Brundin et al., 1994). However, soon after the initial observations in rodents demonstrating a good or even complete restoration of some aspects of the behavioral syndrome, such as rotational asymmetry (Björklund and Stenevi, 1979; Perlow et al., 1979) and simple sensorimotor neglect (Björklund et al., 1981; Dunnett et al., 1987), it became apparent that there are also clear limitations in the capacity of DA grafts to ameliorate more complex sensorimotor behaviors, as shown in

\footnotetext{
Received April 3, 2001; revised May 29, 2001; accepted June 7, 2001.

This study was supported by grants from the Deutsche Forschungsgemeinschaft (Ni 330/4-1). We warmly thank Dr. A. Brandis and Dr. G. F. Walter at the Institute of Neuropathology (Hannover Medical School) for their continuous support with both expertise and technical facilities. J. Wittek contributed with excellent technical work. We thank Dr. Claudia Grothe and Dr. Christian Winkler for valuable suggestions on this manuscript.

Correspondence should be addressed to Dr. Guido Nikkhah, Neurosurgical Clinic, Nordstadt Hospital, Haltenhoffstr. 41, 30167 Hannover, Germany. E-mail: GNikkhah@compuserve.com.

G. Falkenstein's present address: Department of Neurology, Cologne, Germany.

C. Rosenthal's present address: Department of Hematology and Internal Medicine, Essen, Germany.

Copyright (C) 2001 Society for Neuroscience $\quad 0270-6474 / 01 / 216252-12 \$ 15.00 / 0$
}

studies in rodents (Björklund et al., 1994), monkeys (Annett, 1994; Annett et al., 1994), and humans (Freeman and Widner, 1998; Björklund and Lindvall, 2000). More specifically, numerous attempts have been made to restore skilled forelimb use with DA transplants, which have most often failed to demonstrate any ability of the grafts to improve this complex sensorimotor behavioral deficit (Dunnett et al., 1987; Brundin et al., 1994; Barker and Dunnett, 1999; Dobrossy et al., 2000). For this phenomenon, the poor survival rate and incomplete maturation of the grafted dopaminergic neurons, the ectopic placement, and the incomplete reconstruction of the whole nigrostriatal pathway have been put forward as major arguments (Björklund et al., 1994; Dunnett and Björklund, 1994a; Barker and Dunnett, 1999; Winkler et al., 2000).

However, in studies by Nikkhah et al. (1993) and Winkler et al. (1999), first evidence was provided that DA grafts could reinstate, at least partially, skilled forelimb movements in some animals with previous 6-OHDA lesions. Interestingly, these "recovered" animals did not differ from animals that exhibited no significant recovery in paw use with respect to DA graft survival and extent of striatal reinnervation (Nikkhah et al., 1993; Winkler et al., 1999). From these results and other studies, it became likely that more complex graft-host interactions may govern the restorative plasticity of DA grafts in PD, e.g., in relationship to hemispheric dominance-lateralization and paw preference (so called "handedness") (Dunnett et al., 1987; Cabib et al., 1995; Biddle and Eales, 1996, 1999; Nielsen et al., 1997) and the restoration of dopamine content and metabolism (Glick and Ross, 1981; Pearlson et al., 1984; Schwarting et al., 1987; Cabib et al., 1995; Nielsen et al., 1997).

Therefore, the present study was designed to evaluate the 


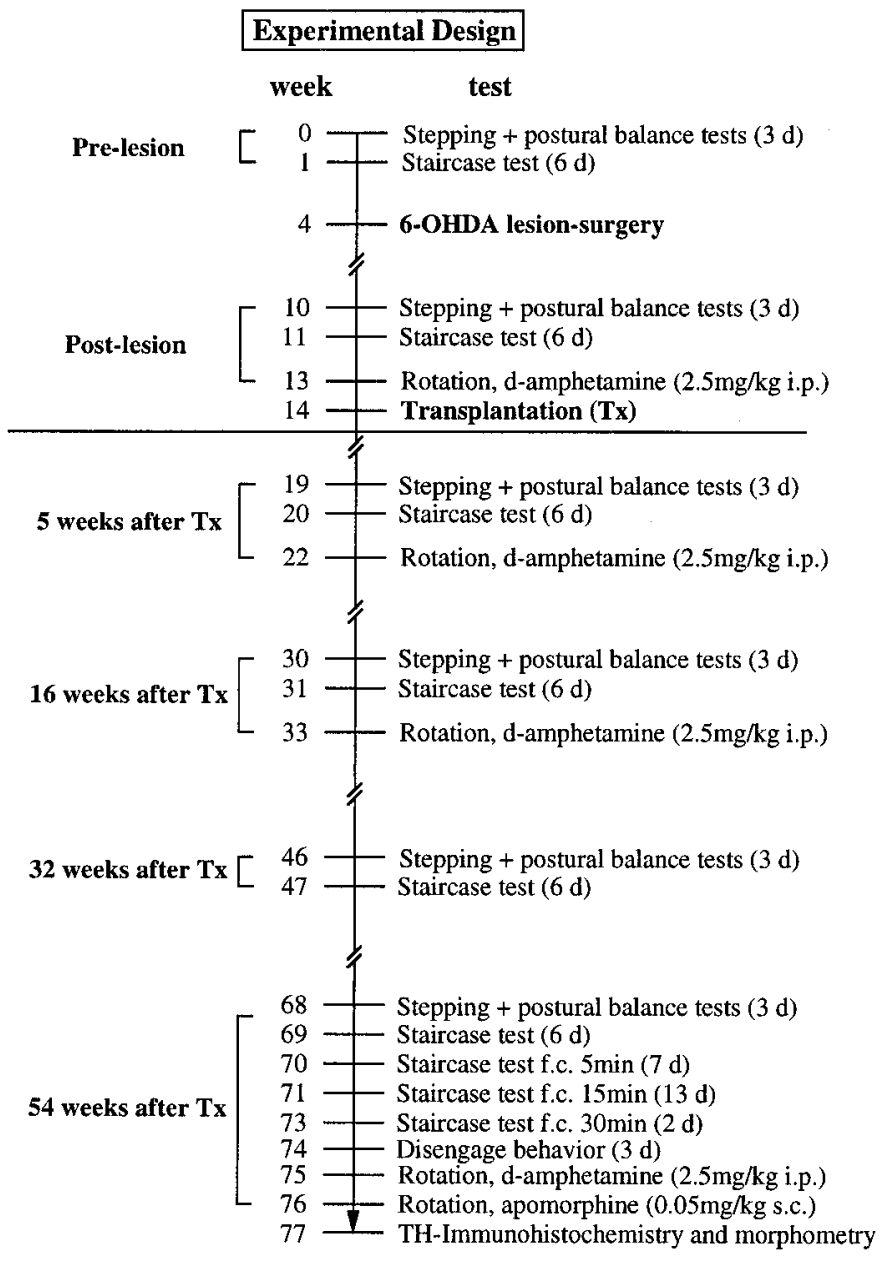

Figure 1. Adult female Sprague Dawley rats $(n=113)$ were tested in the staircase test to determine their handedness (left-handed, $n=43$; righthanded, $n=20$; ambidextrous, $n=50$ ) and in the stepping test before any surgery (data not shown). Then, animals were allocated into three main groups according to their hemispheric dominance, i.e., dominant, nondominant, and indifferent, and subjected to a sequence of surgery and behavioral testing as indicated in this schematic overview.

impact of behavioral lateralization in skilled forelimb use and extensive testing regimes on the plasticity changes induced by unilateral 6-OHDA lesions of the MFB, and ectopically placed intrastriatal DA transplants in adult rats.

\section{MATERIALS AND METHODS}

\section{Experimental design}

One hundred thirteen adult female Sprague Dawley rats $(\mathrm{SD} / \mathrm{Ztm}$; for further information about this strain, see Nikkhah et al., 1998) (200-240 gm body weight at the beginning of the experiment) were used in this study. They were housed under a $12 \mathrm{hr}$ light/dark cycle with ad libitum access to food and water. The exact sequence of the experimental investigations (behavioral tests, surgery, and immunohistochemical analysis) is given in Figure 1. Before lesioning, all animals underwent a paw reaching test to determine the degree of handedness as a measure of hemispheric dominance. Therefore, a coefficient of asymmetry (Cas) was introduced, as described, e.g., by Miklyaeva et al. (1991), and the results of the paw reaching test were entered as follows: Cas $=R-L / R+L$, where $R$ is the number of pellets eaten with the right forelimb and $L$ is the number eaten with the left forelimb. Because the distribution of the results in the number of pellets that were eaten resembled an inverted $U$ shape (Fig. 2), a mean Cas $\pm 25 \%$ of the performance levels (Cas from -0.149 to 0.149 ) was defined as ambidextrous or indifferent (indiff) ( $n=$ 50 rats), a Cas $\leq-0.15$ was defined as left-handed ( $n=43$ rats), and a

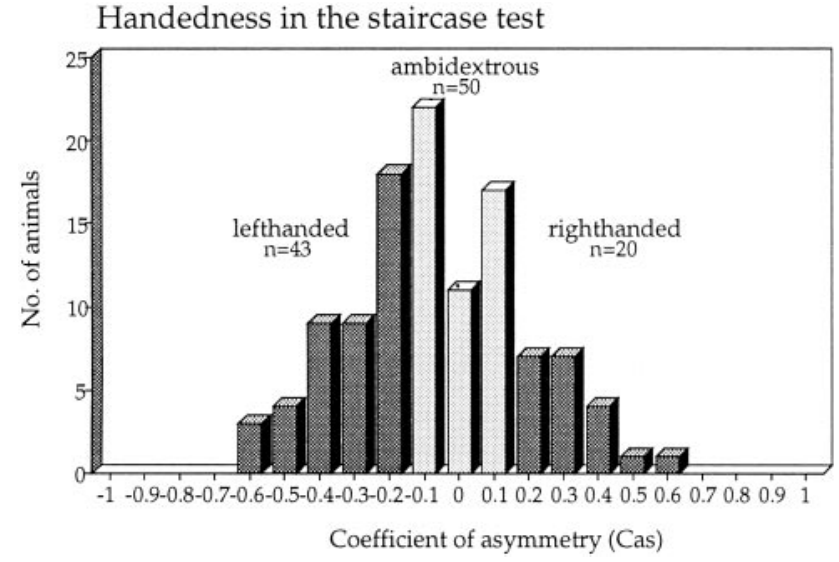

Figure 2. Handedness in the staircase test was tested before any surgery in all animals and was used to determine the level of hemispheric dominance, based on the number of pellets eaten. There appears to be an even and inverted U-shaped distribution within the population basis for skilled forelimb use, with $n=53$ animals demonstrating a clear lateralization of paw preference (left- or right-handed, $-0.15 \leq$ Cas $\geq 0.15$ ) and $n=50$ animals with no paw preference (indifferent, $-0.15 \geq \mathrm{Cas} \leq 0.15$ ).

Cas $\geq 0.15$ was defined as right-handed ( $n=20$ rats). Ten indifferent animals were left as "untreated" normal controls, and all other animals received a unilateral 6-OHDA lesion, either ipsilateral or contralateral to the preferred paw according to their randomly chosen group assignment. Half of the remaining indifferent animals were 6-OHDA-lesioned on the right side of the brain, and the other half on the left side, as shown in Table 1. Eleven animals had to be excluded on the basis of the postlesion rotation test with amphetamine (net rotation $<6$ per minute). Transplantation surgery was performed in 56 6-OHDA-lesioned rats 10 weeks later, followed by a battery of spontaneous and drug-induced behavioral tests, as summarized in Figure 1. Finally, the animals were killed 63 weeks posttransplantation, and the brains were taken for tyrosine hydroxylase $(\mathrm{TH})$ immunohistochemistry and quantitative analysis.

\section{Lesion and transplantation surgery}

Two stereotactic injections of 6-OHDA were made into the right or left mesostriatal dopaminergic pathway at the following coordinates (in $\mathrm{mm}$, with reference to bregma and dura): (1) $2 \mu \mathrm{l}$ of 6-OHDA $(3.6 \mu \mathrm{g} / \mu \mathrm{l}$ 6-OHDA $\mathrm{HBr}$ in $0.2 \mathrm{mg} / \mathrm{ml} \mathrm{L}$-ascorbate-saline) at anteroposterior (AP), -4.0 ; lateral (L), -0.8 ; ventral (V), 8.0 [tooth bar (TB), +3.4]; (2) $2.5 \mu \mathrm{l}$ of 6-OHDA at AP, $-4.4 ; \mathrm{L}, \pm 1.2 ; \mathrm{V}, 7.8(\mathrm{~TB},-2.4)$. The injection rate

\begin{tabular}{|c|c|c|c|}
\hline Group & 6-OHDA lesion & VM graft & $n$ \\
\hline Normal & No & No & 10 \\
\hline Dom H-Les & $\begin{array}{l}\text { Contralateral to the } \\
\text { preferred paw }\end{array}$ & No & 11 \\
\hline Non-dom H-Les & $\begin{array}{l}\text { Ipsilateral to the } \\
\text { preferred paw }\end{array}$ & No & 9 \\
\hline Indiff H-Les & $\begin{array}{l}\text { Right or left } \\
\text { hemisphere }\end{array}$ & No & 17 \\
\hline Dom H-Tx & $\begin{array}{l}\text { Contralateral to the } \\
\text { preferred paw }\end{array}$ & $\begin{array}{l}\text { Contralateral to the } \\
\text { preferred paw }\end{array}$ & 19 \\
\hline Non-dom H-Tx & $\begin{array}{l}\text { Ipsilateral to the } \\
\text { preferred paw }\end{array}$ & $\begin{array}{l}\text { Ipsilateral to the } \\
\text { preferred paw }\end{array}$ & 20 \\
\hline Indiff $H-T x$ & $\begin{array}{l}\text { Right or left } \\
\text { hemisphere }\end{array}$ & $\begin{array}{l}\text { Right or left } \\
\text { hemisphere }\end{array}$ & 16 \\
\hline
\end{tabular}

Seven experimental groups were built on the basis of the handedness in paw preference and performance in the staircase test. Dom, dominant; $\mathrm{H}$, hemisphere; Indiff, indifferent or ambidextrous; Les, 6-OHDA lesion; Tx, 6-OHDA lesioned and transplanted. 
was $1 \mu \mathrm{l} / \mathrm{min}$, and the cannula was left in place for an additional $5 \mathrm{~min}$ before slowly being retracted.

Ten weeks after the 6-OHDA lesion, DA-rich cell suspensions were prepared from ventral mesencephalic tissue of 14-d-old rat fetuses according to a modified version (Nikkhah et al., 1994a, 2000) of the standard cell suspension technique (Björklund et al., 1983). Briefly, the tissue was incubated in $0.1 \%$ trypsin (Worthington, Freehold, NJ), $0.05 \%$ DNase (SigmaDN-25; Sigma, St. Louis, MO), and DMEM at $37^{\circ} \mathrm{C}$ for 20 min, rinsed four times in $0.05 \%$ DNase/DMEM, and mechanically dissociated by trituration through the tip of a $1 \mathrm{ml}$ and then a $200 \mu \mathrm{l}$ Eppendorf pipetteman. Then, the tissue was centrifuged at $600 \mathrm{rpm}$ for $5 \mathrm{~min}$, and the pellet was resuspended in $0.05 \%$ DNase/DMEM. The cell number of this cell suspension was 120,000-140,000 cells per microliter, and the viability was $>95 \%$ before transplantation and $>87 \%$ posttransplantation, as determined by the trypan blue dye exclusion method.

The DA-rich micrografts were implanted using a glass capillary (outer diameter, 50-70 $\mu \mathrm{m}$ ) connected to a $1 \mu \mathrm{l}$ Hamilton microsyringe (Nikkhah et al., 1994a). Two deposits of $0.5 \mu \mathrm{l}$ were placed along each of three implantation tracts in the head of the caudate-putamen, resulting in a total of six micrografts with a total graft volume of $3 \mu \mathrm{l}$ per animal at the following coordinates (TB:0): (1) AP, +1.3; L, 2.8; V, $5.0+4.1 ;$ (2) AP, +0.5; L, 2.3; V, $5.0+4.1$; and (3) AP, +0.5; L, 2.8; V, $5.0+4.1$.

\section{Rotation tests}

Nine weeks after 6-OHDA lesion surgery, the animals were given 2.5 $\mathrm{mg} / \mathrm{kg}$ D-amphetamine (in saline) intraperitoneally, and their rotational behavior was monitored over a $90 \mathrm{~min}$ period in automated "rotometer" bowls according to Ungerstedt and Arbuthnott (1970). Only rats exhibiting a mean net ipsilateral rotation toward the lesion side of at least 6.0 full body turns per minute were included in the study. The amphetamineinduced rotation test was repeated at weeks 22,33 , and 75 of the experiment (Fig. 1). In week 76, the animals were given apomorphine $(0.05 \mathrm{mg} / \mathrm{kg}$, s.c. $)$ in the neck, and their rotational behavior was monitored for $40 \mathrm{~min}$.

\section{Stepping and postural balance tests}

Quantitative movement analysis on each forelimb was performed by using a modified version of the stepping test, as originally described by Schaller et al. (1992), Olsson et al. (1995), and Winkler et al. (1996).

Side-stepping test. Two days before the testing, the animals were handled by the experimenter to become familiar with the different test maneuvers. The actual test was performed twice daily for 3 consecutive days. Briefly, the experimenter held the rat, fixing its hindlimbs with one hand and the forelimb not to be monitored with the other while the unrestrained forelimb was touching the table surface. The number of adjusting steps was counted while the rat was moved sideways along the table with a constant speed $(60 \mathrm{~cm}$ in $6 \mathrm{sec})$, first in the forehand direction and then in the backhand direction. This was done twice daily for each forelimb on 3 consecutive days, and the average of six counts was calculated.

Postural balance test. To evaluate the balancing reaction, the rat was held in the same position as described above, and then the body was tilted along the longitudinal body axis by the experimenter toward the side of the paw touching the table. The counterbalancing reaction of that forelimb was scored from 0 to 3 (Winkler et al., 1996). Briefly, scores were: 0 , representing no detectable muscle reaction in the forelimb; 1 , clear forelimb reaction with muscle contraction, but lack of success in recovering balance; 2 , clear forelimb movement with incomplete recovery of balance and impaired placement of the paw, i.e., the digits were partly crossed over one another; and 3, normal forelimb balancing movement with total recovery of balance comparable with unlesioned controls. Additionally, the "tilt angle" was documented as the angle between the forelimb and the table surface when the rat performed a sideward adjusting step while being rotated as described above $\left(90^{\circ}\right.$, starting point; $0^{\circ}$, endpoint, when the animal was completely rotated without a response). These tests were repeated six times a day during the same session as the side-stepping test on each forelimb for 3 consecutive days, and the final results are expressed as the mean of the $3 \mathrm{~d}$. The test was done prelesion for the evaluation of the "points" and postlesion for the tilt angle in all groups except the indiff hemisphere 6-OHDA lesioned and transplanted (Indiff H-Tx) group, in which the postural balancing reactions were assessed from 16 weeks posttransplantation onward.

\section{Skilled forelimb use (staircase test)}

A modified version of the staircase test (Nikkhah et al., 1998) described by Montoya et al. (1991) was performed prelesion, postlesion, and posttransplantation, as shown in Figure 1. After $2 \mathrm{~d}$ of food deprivation, the animals were tested for 6 consecutive days, except for the 54 weeks posttransplantation test in which the animals were tested for 4 weeks continuously. For each test, the animals were placed into Plexiglas boxes holding a removable double staircase on which steps $2-5$ were baited with 10 food pellets (45 mg; Campden Instruments, Sileby, UK) on each side, and the testing period was $15 \mathrm{~min}$. On day 6 , only one side of the staircase was baited, and the testing period was shortened to $5 \mathrm{~min}$, followed by testing the other side [forced choice test (fc)]. At the 54 week postgrafting time point after 1 week of continuous forced choice tests with a 5 min test period [ $5 \mathrm{~min}$ modified $\mathrm{fc}(\mathrm{mfc})]$, the time interval for testing was extended first to $15 \mathrm{~min}$ per test ( $15 \mathrm{~min} \mathrm{mfc}$ ) for a test period of $13 \mathrm{~d}$ and finally to $30 \mathrm{~min}$ per test $(30 \mathrm{mfc})$ for an additional $2 \mathrm{~d}$. In each test session, several measures were evaluated: the number of pellets eaten (successful reaches), the number of pellets taken (pellets eaten plus pellets grasped but dropped), and the success rate (pellets eaten divided by pellets taken).

Prelesion, the performance in the staircase test was used to define the handedness of each individual rat (Fig. 2).

\section{Disengage behavior}

This test was applied once at 60 weeks after transplantation by an experimenter who was blinded to the animal identity according to the protocol of Schallert and Hall (1988) and Mandel et al. (1990). The perioral region beneath the vibrissae on each side of the head was touched repeatedly by a wooden stick at $1-2 \mathrm{sec}$ intervals while the rats were eating a piece of chocolate. The response latency was measured as the time interval by which a perioral stimulation resulted in an orienting response toward the stimulus or a maximum time of $180 \mathrm{sec}$ if the animal did not respond. The animals were tested once daily on 3 consecutive days. The values given are the means of the three tests.

\section{TH immunohistochemistry}

At 63 weeks postgrafting, the animals were deeply anesthetized with ketamine-rompun and perfused transcardially with $50 \mathrm{ml}$ of $0.9 \%$ saline, followed by $250 \mathrm{ml}$ of ice-cold $4 \%$ paraformaldehyde in $0.1 \mathrm{M}$ phosphate buffer (PB), $\mathrm{pH}$ 7.4. The brains were post-fixed for $12 \mathrm{hr}$ in paraformaldehyde and dehydrated for $24 \mathrm{hr}$ in $20 \%$ sucrose $/ 0.1 \mathrm{M} \mathrm{PB}$. Serial coronal sections ( $30 \mu \mathrm{m}$ thick) were cut on a freezing microtome, and every third section was processed for $\mathrm{TH}$ immunohistochemistry as follows: free floating sections were rinsed three times with $0.2 \mathrm{M} \mathrm{PB}$, quenched with $3 \% \mathrm{H}_{2} \mathrm{O}_{2} / 10 \%$ methanol/PB for $10 \mathrm{~min}$, and rinsed three times with $\mathrm{PB}$ After a preincubation of $1 \mathrm{hr}$ in 5\% normal swine serum (NSS) $/ 0.3 \%$ Triton $\mathrm{X}-100 / \mathrm{PB}$, the sections were incubated with the primary $\mathrm{TH}$ antiserum (diluted 1:500 in 2\% NSS/0.3\% Triton X-100/PB; Pel-Freeze Biologicals, Rogers, AR) overnight at room temperature. After three rinses with $\mathrm{PB}$, sections were incubated with a biotinylated swine-antirabbit IgG (1:200; Dakopatts, Copenhagen, Denmark)/0.3\% Triton $\mathrm{X}-100 / \mathrm{PB}$ for $1 \mathrm{hr}$, rinsed again three times, and transferred to a Vectastain ABC solution/PB for $1 \mathrm{hr}$. The labeling was visualized by a chromogen solution of $0.05 \%$ 3,3-diaminobenzidine and $0.01 \% \mathrm{H}_{2} \mathrm{O}_{2}$. Sections were mounted onto chromalum-coated slides, dehydrated in ascending alcohol concentrations, and coverslipped in DPX.

TH-immunoreactive graft-derived neurons in the striatum were counted microscopically under bright field illumination using $10 \times$ magnification, and an approximation of the total graft cell number was calculated according to the formula of Abercrombie (1946). Graft volumes were determined according to the Cavalieri principle (Gundersen et al., 1988; Mayhew, 1992) with the help of a computerized stereology system equipped with the GRID software (MedicoSoft, Copenhagen, Denmark).

\section{Statistical analysis}

Results are expressed as means \pm SEM. For statistical evaluation, data were subjected to one-way ANOVA and post hoc Bonferroni-Dunn test. There was no significant difference between left- and right-handed animals (whether lesioned-only or lesioned and grafted) with regards to the absolute performance scores in the different behavioral tests. Therefore, 


\section{A. Dom H-Tx}

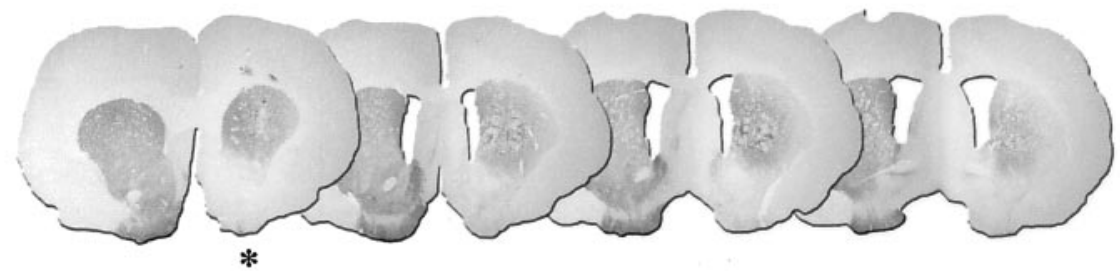

B. Non-dom H-Tx

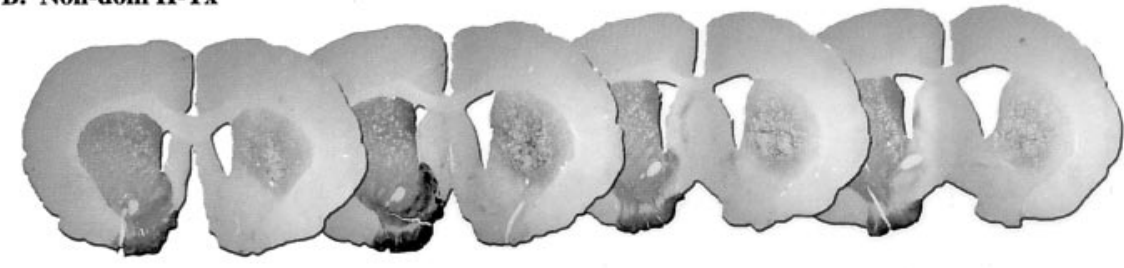

C. Indiff $\mathbf{H}-\mathbf{T x}$

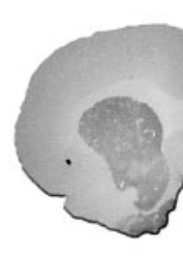

AP:

$+1.3$
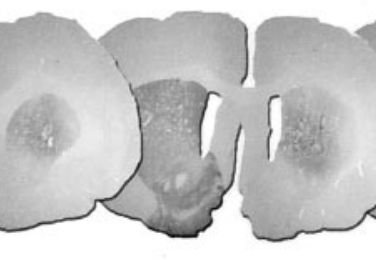

$+\mathbf{1 . 0}$

left- and right-handed animals were pooled into larger groups according to Table 1: dom, for dominant hemisphere, i.e., treatment of the contralateral hemisphere in relation to the left- or right-handed preference in skilled forelimb use; non-dom, for non-dominant hemisphere, i.e., treatment of the ipsilateral hemisphere in relation to the forelimb preference; and indiff, for treatment of one hemisphere in animals with no clear preference in the skilled forelimb test. Statistical significance level was set at $p<0.05$.

\section{RESULTS}

\section{Distribution of handedness and hemispheric dominance}

Hemispheric dominance was defined on the basis of the distribution of handedness in the staircase test. All animals were tested prelesion to identify the degree of forelimb preference; the results are shown in Figure 2. When applying the Cas (see Materials and Methods) for the number of pellets eaten, 43 rats $(38.1 \%)$ were left-handed (Cas $\leq-0.15), 20$ rats $(17.7 \%)$ were right-handed (Cas $\geq 0.15)$, and 50 rats $(44.2 \%)$ were indifferent (ambidextrous). The distribution reveals an almost regular inverted $U$ shape with no clear evidence of a predominance of one forelimb or one hemisphere in this task. The animals were divided then into lesion plus graft, lesioned-only (H-Les), and normal control groups, as shown in Table 1.

\section{TH-positive graft survival}

After the 6-OHDA MFB-lesion, dopaminergic micrografts were implanted as two $0.5 \mu \mathrm{l}$ deposits along three needle tracts in the head of the caudate-putamen unit in the dom H-Tx $(n=19)$, non-dom H-Tx $(n=20)$, and indiff H-Tx $(n=16)$ graft groups. The two graft deposits in each needle tract had fused to form an elongated graft with most of the surviving TH-positive neurons scattered throughout the graft. Abundant TH-positive processes extended into the host neuropil, giving rise to a dense THpositive terminal network reinnervating the head of the striatum (Fig. 3), similar to previous studies using the microtransplantation
Figure 3. TH-immunostained sections illustrating the extent of graft-derived reinnervation after the implantation of dopaminergic grafts along three implantation tracts into the head of the caudate-putamen unit $(*$ indicates right side) for $\operatorname{dom~H-Tx~}(A)$, non-dom H-TX $(B)$, and indiff H-Tx animals $(C)$. Note that the graft-derived TH-positive fiber outgrowth in all three graft groups covers most of the head of the striatum on the right 6-OHDA lesioned side, whereas other areas including the nucleus accumbens, olfactory tubercle, and the most caudal parts of the striatum receive no or only very modest TH-positive reinnervation.

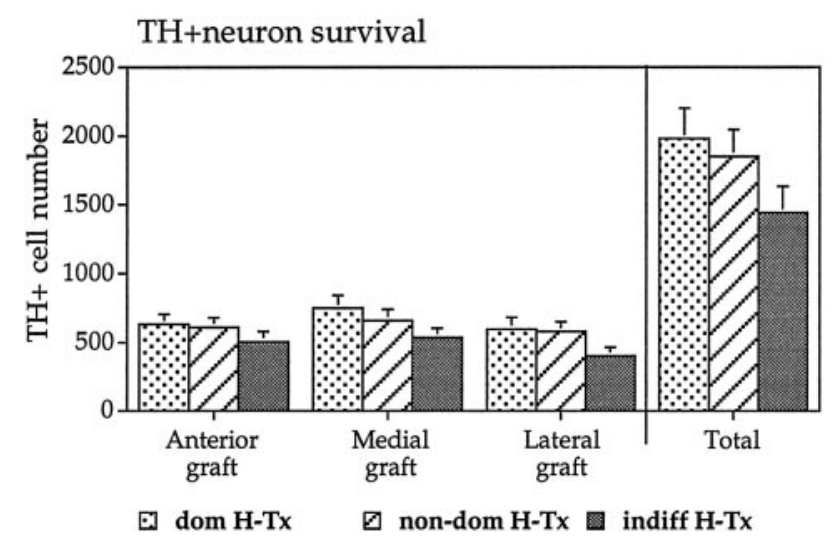

Figure 4. Number of TH-positive cells in the three graft groups expressed as mean \pm SEM. On the left, the mean survival rate for the three implantation sites (anterior, medial, and lateral caudate-putamen) is shown; on the right, the mean survival of TH-positive cells per group is shown. There was no significant difference between the actual implantation locations or between any of the three graft groups (NS).

approach (Nikkhah et al., 1994a,b, 2000; Winkler et al., 1999). However, because of the limited number (3) of implantation tracts placed in the center of the caudate-putamen unit in the present study, as compared with previous studies (6-7) (Nikkhah et al., 1994b, 2000; Winkler et al., 1999), the nucleus accumbens and more caudal parts of the striatum did not receive a significant graft-derived reinnervation. The mean total number of surviving TH-positive cells per graft was $1980 \pm 222$ cells in the dom H-Tx group, $1852 \pm 195$ cells in the non-dom H-Tx group, and $1442 \pm$ 190 cells in the indiff H-Tx group, with no significant difference between those three groups or between the anterior, medial, and lateral graft location (Fig. 4). 


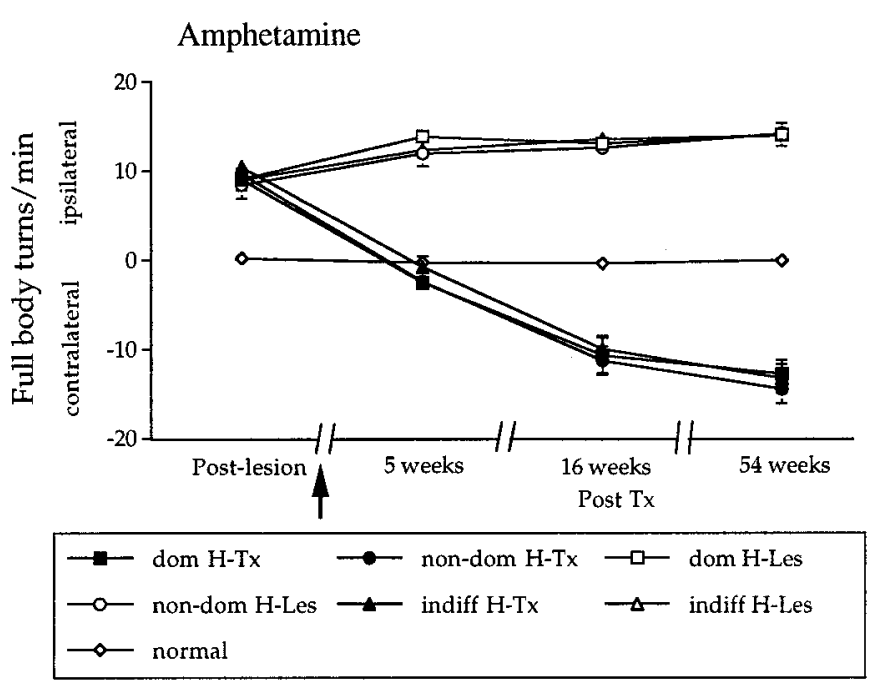

Figure 5. Amphetamine-induced rotation $(2.5 \mathrm{mg} / \mathrm{kg}$, i.p.) postlesion and 5,16 , and 54 weeks posttransplantation. At 5 weeks postgrafting, there was already a complete amelioration of amphetamine-induced rotational asymmetry in all three graft groups. At 16 and 54 weeks posttransplantation, all graft groups exhibited a strong contralateral turning response. All three graft groups were significantly improved at all time points postgrafting, as compared with their respective lesion-only control group ( $p<0.001$ ), with no significant difference between the lesion or graft groups. Arrow indicates time point of transplantation.

\section{Drug-induced rotation Amphetamine}

Unilateral 6-OHDA lesions induced a rotational asymmetry from a mean of 8.4 (non-dom H-Les) to 10.4 (indiff H-Tx) full body turns per minute ipsilateral to the lesion (Fig. 5). Already 5 weeks postgrafting, there was a significant and complete restoration of rotational asymmetry in all grafted groups $(p<0.01)$. At 16 and 54 weeks posttransplantation, the grafted groups had uniformly reversed their turning behavior to $>10$ full body turns per minute contralateral to the lesion, with no significant difference between the graft groups. In contrast, all lesion-only groups (dom H-Les, non-dom H-Les, indiff H-Les) showed a strong and consistent ipsilateral amphetamine-induced rotational response over the entire test period, whereas normal animals showed no rotational asymmetry.

\section{Apomorphine}

In the apomorphine-induced rotation test at 76 weeks (Fig. 6), there was a graft-induced reduction of $\sim 70 \%$ in the three grafted groups as compared with the lesion-only groups $(p<0.001)$, which was still significantly above normal levels.

\section{Side-stepping test}

\section{Forehand}

First, all animals underwent the stepping test, even before the staircase test, before lesion and transplantation surgery (Fig. 7A). The level of performance in the forehand direction prelesion varied from a mean of $6.1 \pm 1.0$ adjusting steps in the dom H-Les group to $9.1 \pm 0.8$ adjusting steps in the indiff H-Les group. Interestingly, animals that exhibited a similar degree of performance level in the staircase test before surgery (dom H-Tx vs dom H-Les, see below), showed a statistically significant difference $(8.6 \pm 0.5$ vs $6.1 \pm 1.0 ; p<0.02)$ prelesion. The 6 -OHDA lesion induced significant and long-lasting impairments $(<1$ adjusting step) in all three lesion-only groups with no signs of

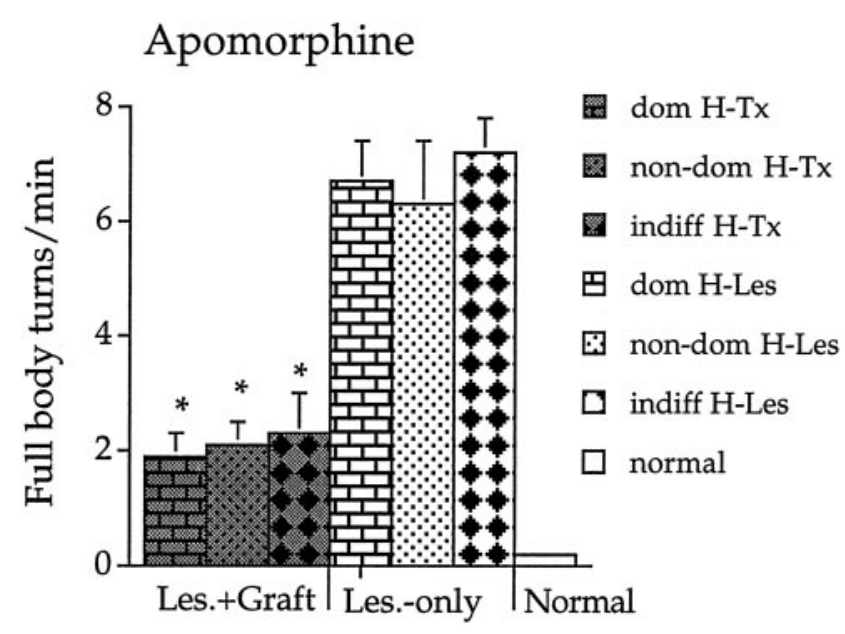

Figure 6. Apomorphine-induced rotation $(0.05 \mathrm{mg} / \mathrm{kg}$, s.c. $)$ at 76 weeks posttransplantation. All transplanted groups show a substantial and highly significant reduction $(-70 \%)$ in rotational asymmetry. *, Significant difference from the respective lesion-only group; $p<0.001$.

spontaneous recovery up to 54 weeks postlesion as compared with the normal controls $(p<0.001)$. All transplanted groups showed a similar significant improvement 5 weeks posttransplantation, with $3.6 \pm 0.5$ steps for the dom H-Tx, $4.3 \pm 0.7$ steps for the non-dom H-Tx, and $4.7 \pm 0.8$ steps for the indiff H-Tx group compared with their respective lesion-only controls $(p<0.001)$, but still significantly below normal control values $(p<0.05)$. However, this significant recovery in forehand stepping decreased clearly after 16 weeks postgrafting and only remained significantly above lesion-only performance levels for the indiff H-Tx groups at 32 and 54 weeks postgrafting $(p<0.05)$, whereas the other two graft groups were no longer significantly different from their respective lesion-only groups at the 16, 32, and 54 week time points.

\section{Backhand}

Side-stepping movements in the backhand direction were less severely, although significantly, decreased after the unilateral 6-OHDA lesion (Fig. 7B). The number of adjusting steps postlesion was reduced in the range of $21 \%$ in the non-dom H-Les group $(9.6 \pm 1.2)$ to $52 \%$ in the dom H-Tx group $(5.4 \pm 0.8)$ and remained significantly impaired over the entire testing period in all lesioned and transplanted groups when compared with normal control animals $(p<0.01)$. Again, 5 weeks after transplantation surgery, there was a significant $(p<0.05)$, although partial, improvement in all grafted groups to performance levels between lesion-only and normal animals, with no significant difference between the transplanted groups; this persisted throughout the 1 year testing period. At 54 weeks posttransplantation, the performance level reached $9.4 \pm 0.7$ adjusting steps for the dom H-Tx group, $9.9 \pm 0.6$ adjusting steps for the non-dom H-Tx group, and $10.0 \pm 0.6$ adjusting steps for the indiff H-Tx group.

\section{Postural balance test}

All animals counteracted the loss of balance adequately with both forelimbs when tilted sideward prelesion. The performance score reached maximum levels of 17-18 points, out of 18 points in all groups, as shown for the contralateral forelimb (with respect to the side of later surgery) in Figure $8 A$ (animals from the indiff H-Tx group were tested from week 16 posttransplantation onward). The balancing reaction was almost completely lost after 
A Stepping: Forehand
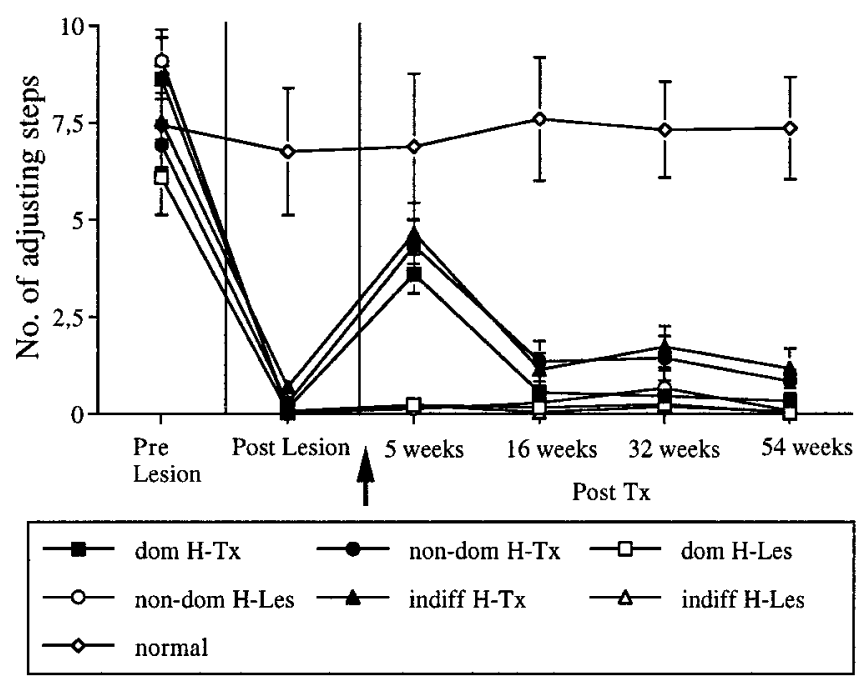

(B) Stepping: Backhand

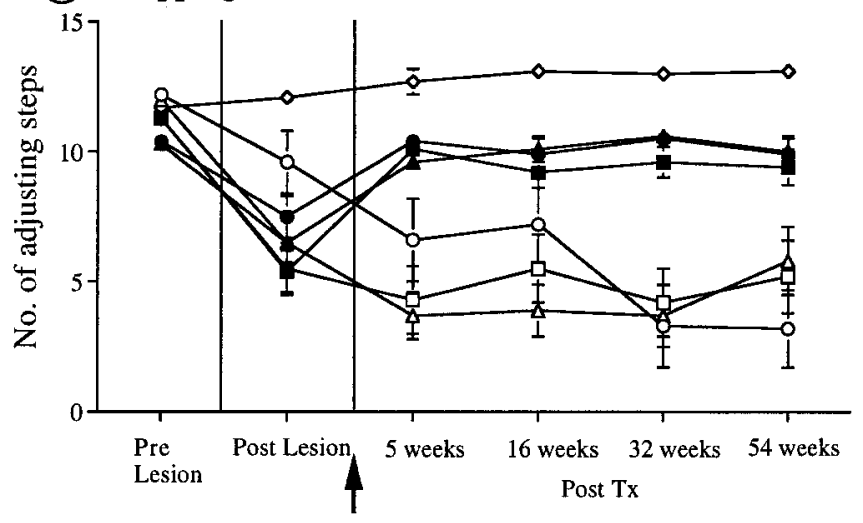

Figure 7. The number of adjusting steps in the forehand $(A)$ and backhand $(B)$ direction are shown prelesion, postlesion, and over a 1 year time course posttransplantation. There was a significant drop in performance level after the 6-OHDA lesion, which was partially restored by the intrastriatal nigral grafts placed into the dominant, non-dominant, or indifferent hemisphere. Arrows indicate time point of transplantation.

the 6-OHDA lesion in all groups that were tested and remained stable in the lesion-only groups, although there was a slight trend for spontaneous recovery at the end of the testing period in the lesioned animals $(4.6 \pm 2.1$ for dom H-Les, $2.1 \pm 1.6$ for non-dom H-Les, $7.0 \pm 1.9$ for indiff $\mathrm{H}$-Les at 54 weeks). Five weeks after transplantation surgery, there was already a clear improvement of balancing reaction in all grafted groups $(p<0.01$; graft groups vs lesion-only), which continued until 1 year postgrafting with final mean performance score (54 weeks) of $12.1 \pm 1.4$ for the dom H-Tx animals, $14.4 \pm 1.1$ for non-dom H-Tx animals, and $15.4 \pm$ 1.2 for indiff $\mathrm{H}-\mathrm{Tx}$ animals ( $p>0.05$; NS between graft groups). Interestingly, the scores of the animals from the indiff H-Tx group were in almost exactly the same range as the other two grafted groups at 16, 32, and 54 weeks posttransplantation, indicative of a primary functional graft-host interaction not significantly influenced by prelesion and/or posttransplantation training. Additionally, at 54 weeks posttransplantation, there was no more significant difference between the indiff H-Tx and non-dom H-Tx and the normal groups $(p>0.05$, NS), indicative of a complete restoration of this aspect of the postural balancing reaction.

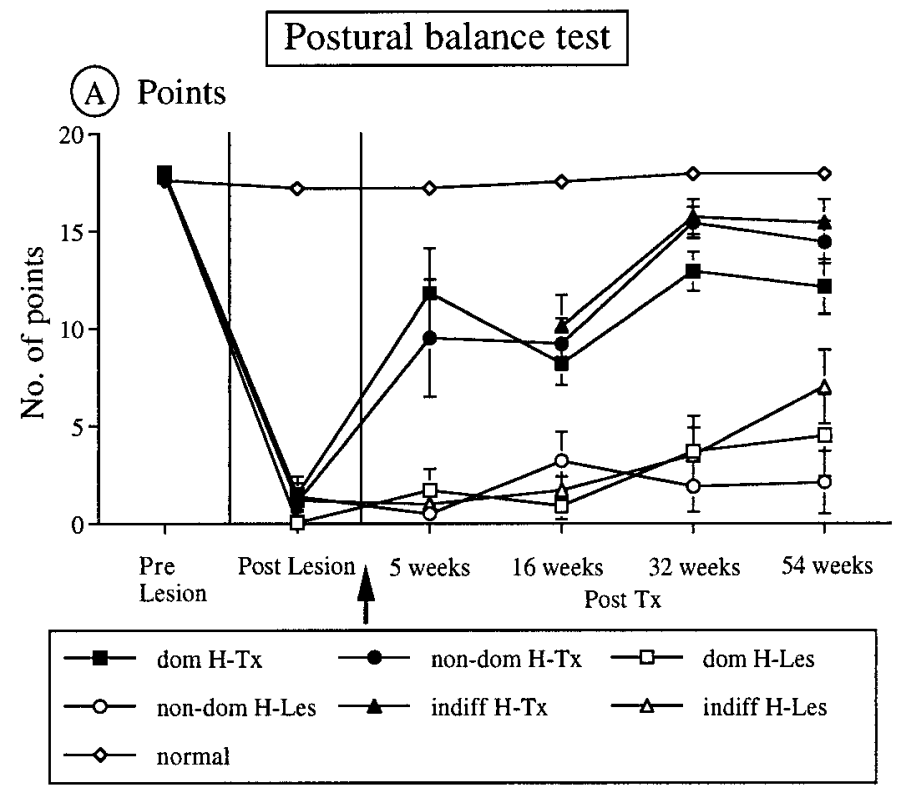

(B) Tilt angle

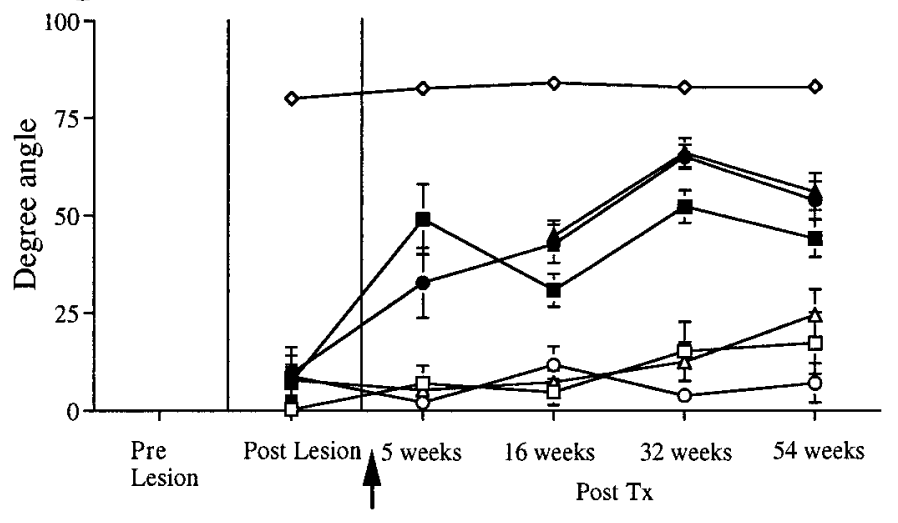

Figure 8. Postural balancing reactions were measured as the quality of paw placement $(A)$ and the tilt angle of movement initiation of the forelimb $(B)$. There is an immediate graft-induced functional recovery in both parameters already at the 5 week posttransplantation time point, which remained stable over the entire 1 year time period. Note that indiff $\mathrm{H}-\mathrm{Tx}$ was tested first at 16 weeks postgrafting but exhibited a degree of functional recovery similar to the dom H-Tx and non-dom H-Tx groups. Arrows indicate time point of transplantation.

The analysis of the tilt angle when the counteracting balancing reaction was initiated demonstrates a similar time-dependent development of lesion-induced deficits and graft-derived recovery as seen for the quality of paw placement above (Fig. $8 B$ ). After the 6-OHDA lesion balancing reactions in the contralateral forelimb were initiated very late at an angle in the range of $0.3 \pm$ $0.3^{\circ}$ (dom H-Les) to $9.5 \pm 6.8^{\circ}$ (non-dom H-Tx) compared with $79.4 \pm 2.9^{\circ}$ in normal controls $(p<0.001)$. At 5 and 16 weeks postgrafting, there was a partial but significant recovery in the grafted groups that further improved at 32 and 54 weeks $(44.1 \pm$ $4.7^{\circ}$ dom H-Tx, $53.8 \pm 4.8^{\circ}$ non-dom H-Tx, and $56.0 \pm 4.8^{\circ}$ indiff H-Tx) compared with lesion-only animals $(p<0.05)$. The lesiononly animals leveled off at a maximum tilt angle of $25^{\circ}$ at the end of the test period at 54 weeks. In contrast to the performance scores of paw placement during the balancing reaction (points, see above) graft-induced functional improvement for the tilt angle did not reach normal levels throughout the whole testing 


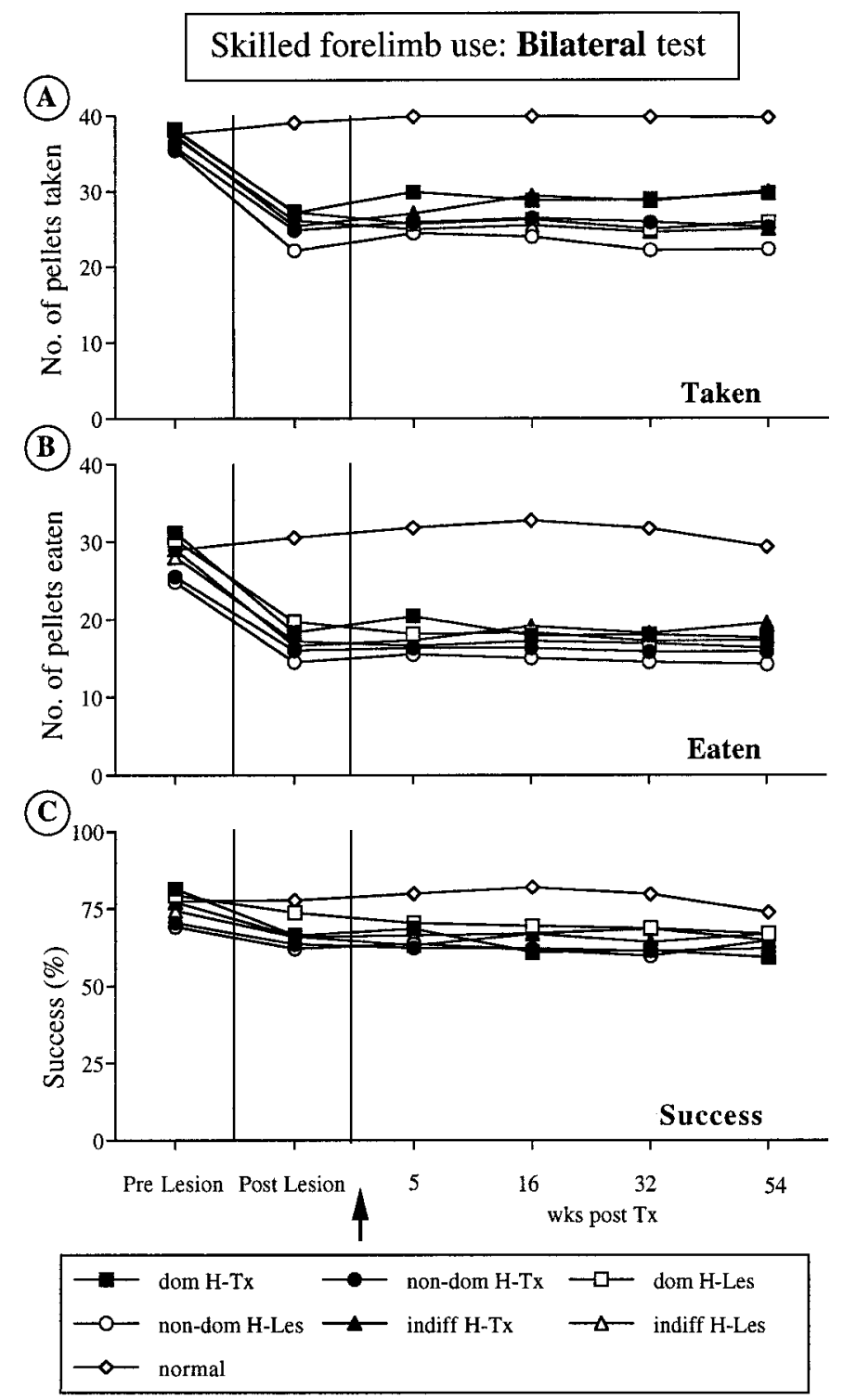

Figure 9. Skilled forelimb use as assessed in the bilateral staircase test. The mean number of pellets taken $(A)$ and eaten $(B)$ and the success rate $(C)$ are given, when both sides (left and right) of the staircase were baited with 40 pellets each and the test was run for $15 \mathrm{~min}$. The 6-OHDA lesion induced a substantial impairment in the number of pellets taken and eaten in all groups compared with normal controls $(p<0.001)$, which remained stable over the entire 1 year testing period. No significant graft-induced functional effects were observed during the bilateral test. Arrow indicates time point of transplantation.

period. Overall, there was no significant difference between the graft groups at any of the time points.

\section{Skilled forelimb use (staircase test) Bilateral test}

When 40 food pellets were provided simultaneously on both sides of the staircase for $15 \mathrm{~min}$, there was no significant difference in the number of pellets taken prelesion (contralateral to the later surgery) between the seven experimental groups tested (Fig. 9A). The performance reached a mean level of between 37 and 38 pellets taken in all groups. The unilateral 6-OHDA lesion induced a significant impairment of $\sim 29-42 \%$ in the number of pellets taken in all lesioned groups, which remained stable in the lesion-only groups over the entire 54 week test period (number of pellets taken at 54 weeks, $25.6 \pm 1.4$ for dom H-Les; $21.8 \pm 2.8$ for non-dom H-Les; $24.3 \pm 1.2$ for indiff H-Les). The three transplanted groups did show some tendency for a recovery in the bilateral test (number of pellets taken at 54 weeks, $29.7 \pm 1.3$ for dom H-Tx; $25.6 \pm 1.4$ for non-dom H-Tx; $29.8 \pm 1.4$ for indiff H-Tx), but this failed to reach significance with respect to their respective lesioned group or in comparison to their own postlesion and pretransplantation scores $(p>0.05)$. Similarly, the bilateral test revealed a substantial lesion-induced deficit in the number of pellets eaten, starting with the following prelesion scores (number of pellets eaten): normal, $28.5 \pm 1.0$; non-dom H-Les, $27.9 \pm 1.7$; non-dom H-Tx, $27.1 \pm 1.0$; indiff H-Les, $28.4 \pm 1.5$; indiff H-Tx, $30.1 \pm 0.9$; dom H-Les, $31.6 \pm 1.1$; dom H-Tx, $32.2 \pm 0.9$ (significant difference between dom H-Tx and non-dom H-Tx, $p<0.01)$. The number of pellets eaten significantly decreased to a mean of $14-17$ pellets postlesion (42-50\% at 54 weeks, $p<0.001$ ), as shown in Figure 9B. A significant recovery in the number of pellets eaten was not observed in any of the three transplanted groups in the affected forelimb during the entire bilateral testing period. The success rate was less severely affected and fell from a mean $72-83 \%$ before the lesion to $62-74 \%$ after the unilateral 6-OHDA lesion (Fig. 9C), although this impairment only reached significance in the transplanted groups dom H-Tx $(59.3 \pm 2.8 \%$, at 54 weeks; $p<0.001)$ and non-dom H-Tx $(62.3 \pm 1.9 \%$, at 54 weeks; $p<0.001)$ as compared with normal $(77.2 \pm 1.7 \%$, at 54 weeks $)$, whereas the other groups demonstrated a non-significant reduction of $\sim 10$ $12 \%$ in the success rate with no signs of graft-induced recovery in the indiff $\mathrm{H}-\mathrm{Tx}$ group $(64.9 \pm 3.1 \%$; NS).

\section{"Standard" unilateral forced choice test}

At day 6 of each test session, only one side of the staircase contralateral to the affected forelimb was baited with 40 pellets, and the animals were tested during $5 \mathrm{~min}$ (prelesion until 54 weeks) (Fig. 10). After the 6-OHDA lesion, a substantial impairment in the number of pellets taken (prelesion, 36-39 pellets taken) was observed in all lesioned groups (Fig. 10 $A$ ): $24.1 \pm 2.1$ for dom H-Les, $21.6 \pm 2.0$ for non-dom H-Les, $22.4 \pm 1.2$ for indiff H-Les, as compared with $39.7 \pm 0.2$ pellets taken for normal controls $(p<0.001)$. Repeated test sessions at 5, 16, 32, and 54 weeks (Fig. 10) did not reveal any significant improvement in any of the three lesion-only groups. In contrast, the indiff H-Tx group did show a significant improvement in the level of performance starting at 16 weeks postgrafting $(31.1 \pm 1.1$ pellets taken), when compared with both their postlesion scores $(23.3 \pm 1.6$ pellets taken; $p<0.01)$ and their respective indiff H-Les control group at 16 weeks $(22.6 \pm 1.1$ pellets taken; $p<0.001)$, as shown in Figure $10 A$. This significant improvement of the indiff H-Tx group remained stable at 32 weeks $(30.8 \pm 0.8$ pellets taken) and 54 weeks $(31.3 \pm 1.1$ pellets taken $)$ postgrafting. Although there appears to be a trend for recovery in the other two transplanted groups, the differences did not reach statistical significance in comparison with their respective control values during the repeated test sessions of the standard forced choice test (Fig. 10 $\mathrm{A}$ ). A rather similar development is seen for the number of pellets eaten in the unilateral skilled forelimb test (Fig. 10B). The 6-OHDA lesion induced a marked behavioral impairment in all groups, which remained unaffected by repeated testing and training in the lesion-only groups during the entire test period, leveling off at 54 weeks at $13.5 \pm 1.7$ pellets eaten for dom H-Les, $12.0 \pm 2.0$ pellets eaten for non-dom $\mathrm{H}$-les, and $14.8 \pm 1.6$ pellets 


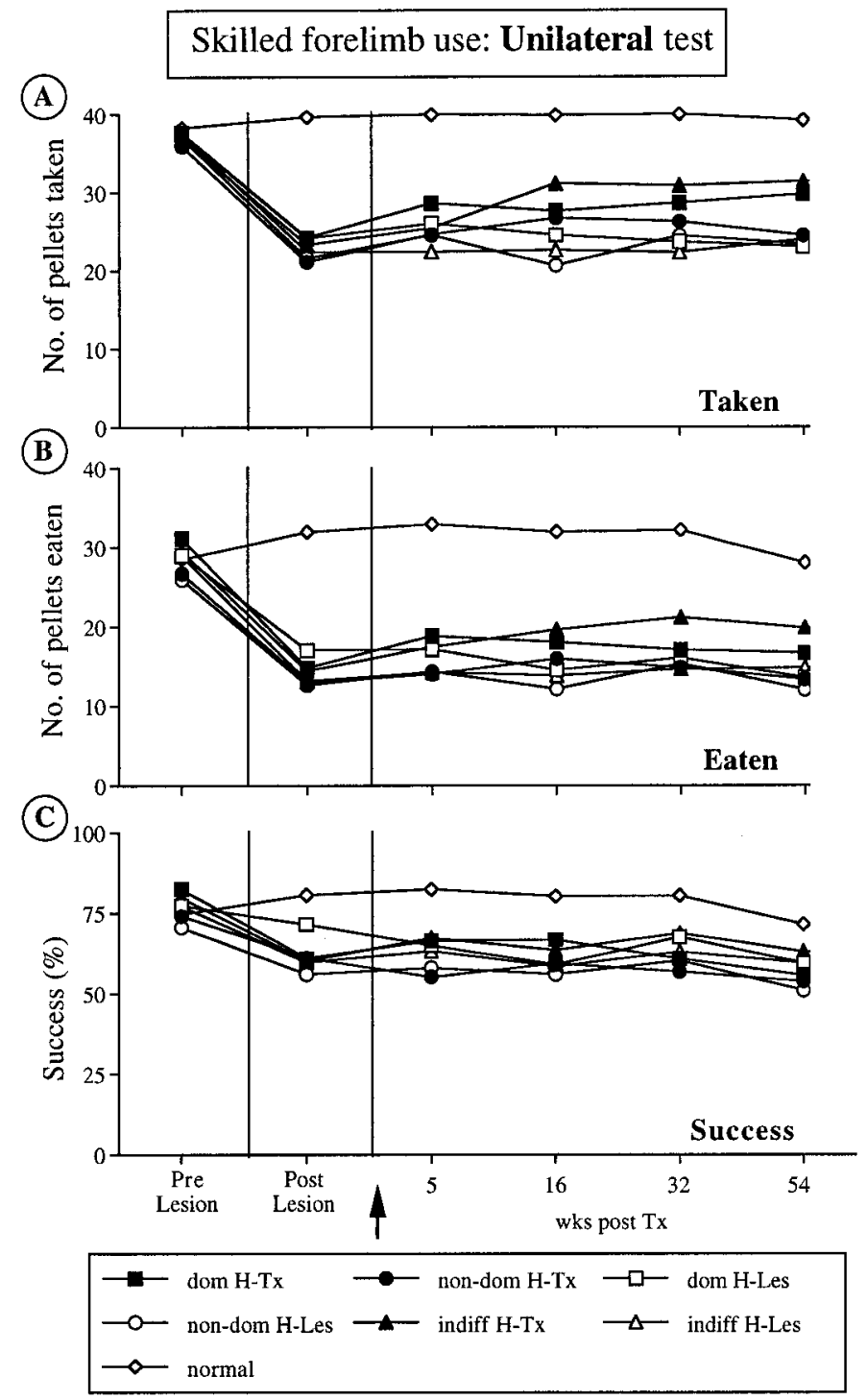

Figure 10. Skilled forelimb use as assessed in the standard unilateral forced choice staircase test (only the contralateral side baited with 40 pellets) providing the mean number of pellets taken $(A)$ and eaten $(B)$ and the success rate $(C)$ during the standard test condition $(5 \mathrm{~min})$. Note that a significant improvement was seen only in the indiff $\mathrm{H}$-Tx group for the number of pellets taken and eaten at 16 weeks posttransplantation, which remained stable over the further testing period. Arrow indicates time point of transplantation.

eaten for indiff H-Les ( $p<0.001$, compared with normal). Interestingly, no significant transplant-induced recovery was seen at 5 weeks postgrafting, whereas at 16 weeks postgrafting during the second testing period animals from the indiff H-Tx group increased the number of pellets eaten significantly to $19.6 \pm 1.3$ pellets compared with $13.8 \pm 1.4$ in indiff H-Les animals $(p<$ 0.01 ), and the improvement in this group remained stable to 32 weeks posttransplantation $(21.1 \pm 1.2$ vs $14.6 \pm 1.5 ; p<0.001)$, but failed to reach significance at 54 weeks $(19.8 \pm 1.4$ vs $14.8 \pm$ $1.6, p>0.05)$. In contrast, dopaminergic transplantation in the dom H-Tx and non-dom H-Tx groups did not result in a significant improvement of lesion-induced deficits in the number of pellets eaten, in comparison either to their own postlesion scores or to their respective control group $(p>0.05)$. Furthermore, grafted animals from the indiff $\mathrm{H}$-Tx group ate significantly more pellets than animals from the non-dom H-Tx group at the 32 and 54 week time points $(p<0.01)$. The results of the success rate are illustrated in Figure $10 \mathrm{C}$ in which the 6-OHDA lesion induced a mild (e.g., $71.2 \pm 4.7 \%$ in the dom H-Les group; NS, compared with normal) to moderate $(59.6 \pm 4.3 \%$ in the indiff H-Les group, $p<0.005)$ impairment compared with normal controls $(79.4 \pm$ $3.4 \%$ ) with no clear evidence for graft-induced functional restoration throughout the whole testing period.

\section{"Modified" unilateral forced choice test}

During the modified forced choice test, continuous testing and training was performed only of the contralateral paw (in relation to the side of surgery) for a further period of $7 \mathrm{~d}$ for 5 min test sessions ( $5 \mathrm{~min} \mathrm{mfc}$ ), $13 \mathrm{~d}$ for $15 \mathrm{~min}$ test sessions (15 $\mathrm{min} \mathrm{mfc}$ ), and $2 \mathrm{~d}$ for $30 \mathrm{~min}$ test sessions (30 min $\mathrm{mfc}) 1$ year after transplantation; the results are illustrated in Figure 11. Continuous training and testing using the same testing time period (5 $\mathrm{min}$ ) did not result in any further improvement in the groups tested, as compared with the results of the standard forced test (5 min fc) (Fig. 11A). The indiff H-Tx group further increased its performance level when the individual test interval was prolonged to $15 \mathrm{~min}(35.7 \pm 0.8$ pellets taken $)$ and $30 \mathrm{~min}(37.2 \pm 0.7$ pellets taken) per session (Fig. 11A), whereas no such development was seen in any of the lesion-only groups. During the forced choice tests, the performance level of the indiff H-Tx group was no longer significantly different from normal intact controls (39.6 \pm 0.1 pellets taken). The dom H-Tx group demonstrated a first significant improvement to a performance level of $34.1 \pm 1.2$ pellets taken, as compared with $23.4 \pm 2.0$ pellets taken for the dom H-Les group $(p<0.001)$, when the test interval was extended to $15 \mathrm{~min}$ (Fig. 11 $\mathrm{A}$ ). A similar result was obtained for the 30 min test interval (dom H-Tx, $33.9 \pm 1.5$ pellets taken, vs dom H-Les, $24.9 \pm 2.5$ pellets taken; $p<0.01$ ). Again, the performance of the dom H-Tx group at these two latter time points was not significantly different from normal controls. Finally, animals in the non-dom H-Tx group remained at the performance level of the lesion-only animals until the last two tests during the modified forced choice test but demonstrated a statistically significant difference from their lesion control group (non-dom H-Les) at the 30 min test, at which they took $31.9 \pm 1.8$ pellets as compared with $22.8 \pm 3.7$ pellets in the non-dom H-Les group $(p<0.01)$. This was also non-significantly different from normal controls. Interestingly, the performance level of the indiff H-Tx group was significantly above the non-dom H-Tx group at the 54 week ( 5 $\min \mathrm{fc} ; p<0.01)$ and $5 \mathrm{~min} \operatorname{mfc}$ tests $(p<0.001)$.

The number of pellets that were eaten (Fig. 11B) also remained substantially impaired in the lesion-only groups during the modified forced choice tests: $15.8 \pm 2.5$ pellets for the dom H-Les group, $12.2 \pm 2.4$ pellets for the non-dom H-Les group, and $13.3 \pm 1.6$ pellets for the indiff H-Les group, at the final $30 \mathrm{~min}$ modified forced choice test. Training and repeated testing did not enhance the significant, but partial recovery seen in the indiff $\mathrm{H}$-Tx group during the $5 \mathrm{~min} \mathrm{mfc}$ test. A further significant increase in the number of pellets eaten was observed during the $15 \min (23.9 \pm 1.4$ pellets $)$ and $30 \mathrm{~min}(24.5 \pm 1.4$ pellets $)$ forced choice tests, which was non-significantly different from normal controls $(31.3 \pm 1.4$ pellets, $30 \mathrm{~min} \mathrm{mfc})$. Performance of the indiff H-Tx group during the $5 \mathrm{~min} \mathrm{fc}$ and $15 \mathrm{~min} \mathrm{mfc}$ tests was significantly above the non-dom H-Tx group $(p<0.01)$. The dom H-Tx group achieved significantly above lesion-only (dom H-Les) scores in the number of pellets eaten in the $15 \mathrm{~min} \mathrm{mfc}$ test $(21.6 \pm 1.2$ vs $14.2 \pm 2.2 ; p<0.01)$ and a clear trend toward 

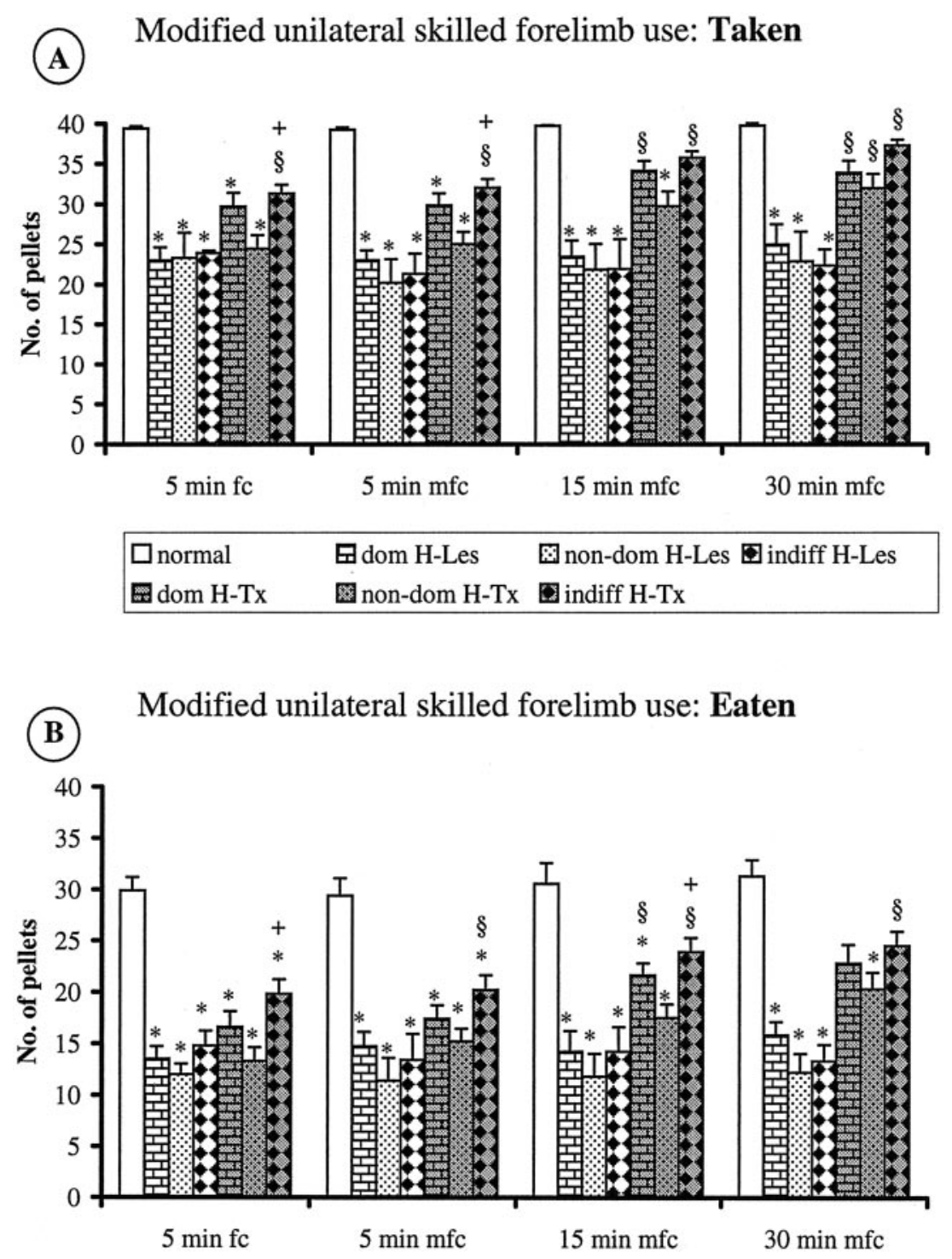

Figure 11. The results of the modified forced choice test for skilled forelimb use are illustrated for the number of pellets taken $(A)$ and eaten $(B)(5$ min fc tested for $1 \mathrm{~d}$ at 54 weeks followed by $5 \mathrm{~min}$ mfc tested for $7 \mathrm{~d}$ continuously, $15 \mathrm{~min} \mathrm{mfc}$ tested for $13 \mathrm{~d}$ continuously, and $30 \mathrm{~min}$ tested for $2 \mathrm{~d}$ continuously). Note, that a significant improvement in skilled forelimb use was seen first in the indiff H-Tx group for the number of pellets taken and eaten at the $5 \mathrm{~min}$ fc test, which further increased during the more extensive training and testing period until the final $30 \mathrm{~min} \mathrm{mfc}$ test. During the latter test periods, also, the dom H-Tx group (in the 15 and 30 min time test) and the non-dom H-Tx (only in the $30 \mathrm{~min}$ time test) demonstrated a significant behavioral recovery in comparison to their respective lesion-only group. * Significant difference from normal, $p<0.001$, ANOVA with post hoc Bonferroni test; + , significant difference from non-dom H-Tx group, $p<0.01$; $\$$, significant difference from respective lesion-only group, $p<0.01$.

improvement in the $30 \mathrm{~min} \mathrm{mfc}$ test $(22.8 \pm 1.8$ vs $15.8 \pm 2.5 ; p>$ $0.05)$; the latter performance was also non-significantly different from normal controls. In contrast, animals from the non-dom H-Tx group failed to demonstrate significant recovery levels in the number of pellets eaten under any of the testing conditions examined and remained significantly below normal control levels throughout the entire continuous testing paradigm $(p<0.001)$.

\section{Disengage behavior}

The unilateral 6-OHDA lesion induced a marked increase in contralateral response latency in the disengage behavior test from $1.1 \pm 0.2 \mathrm{sec}$ in the normal group (Fig. 12) to $134.4 \pm 16.3 \mathrm{sec}$ (indiff H-Les), $153 \pm 19.0 \mathrm{sec}$ (dom H-Les), and $180.0 \pm 0.0 \mathrm{sec}$ (non-dom H-Les), which was highly significant for all lesioned groups $(p<0.001)$. Although there was a clear trend in all transplanted groups toward improvement from $-11.3 \%$ in the indiff H-Tx group, $-14.5 \%$ in the non-dom H-Tx group, up to $-22.2 \%$ in the dom H-Tx group as compared with their lesioned control group, none of these results reached statistical significance. However, the response latency was significantly reduced in the dom H-Tx group as compared with the non-dom H-Tx group $(-26.4 \% ; p=0.027)$.

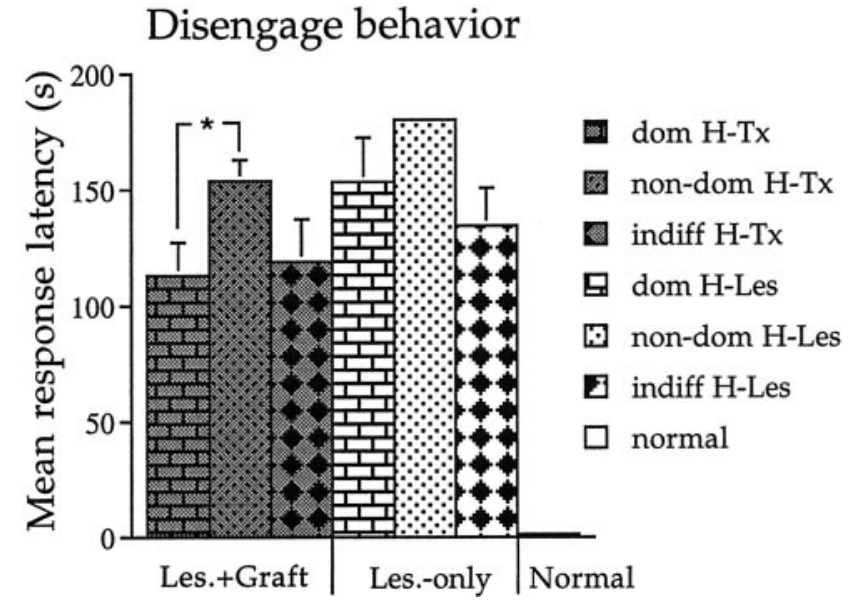

Figure 12. Disengage behavior was tested 60 weeks posttransplantation and revealed a dramatic increase in mean response latency in all lesiononly and graft groups, as compared with normal controls $(p<0.001)$. There was a trend toward a reduced orientation time in the three grafted groups; however, this failed to reach significance $(p>0.05)$. *, Significant difference; ANOVA with post hoc Bonferroni test; $p<0.05$. 


\section{DISCUSSION}

The results of the present study provide evidence, for the first time, that the functional restorative capacity of dopaminergic transplants in PD is significantly governed by the degree of hemispheric dominance (lateralization) and extensive testing regimes in complex sensorimotor behaviors, as demonstrated for skilled forelimb movements. Before lesion surgery, rats were tested in their paw preference and performance level in skilled forelimb movements using the staircase test and subdivided into dominant (dom-H), non-dominant (non-dom $\mathrm{H}$ ), and indifferent (indiff $\mathrm{H}$ ) hemisphere groups based on a coefficient of asymmetry, as previously used by others (Miklyaeva et al., 1991). This was followed by 6-OHDA lesions only or by additional dopaminergic transplants into the respective hemisphere. All three graft groups already demonstrated a rapid and complete restoration of amphetamine-induced rotational asymmetry 5 weeks after transplantation and a $70 \%$ reduction in apomorphine-induced rotation indicative of good graft survival and functional reinnervation. In the stepping and postural balance tests, a significant, although partial long-term improvement was observed among all graft groups, with no correlation to the extent of hemispheric dominance defined by the preference for skilled forelimb use. The 6-OHDA-induced deficits in disengage behavior were not significantly affected by the dopaminergic transplant locations used in the present study, which is in agreement with previous studies (Mandel et al., 1990; Nikkhah et al., 1993). Most importantly and extending previous observations (Dunnett et al., 1987; Montoya et al., 1990; Nikkhah et al., 1993; Abrous et al., 1993a,b; Winkler et al., 1999), the recovery of 6-OHDA-induced deficits in skilled forelimb use was significantly influenced by the degree of hemispheric dominance, which the animals exhibited in paw preference and performance before lesion and transplantation surgery. As may not have been expected, the restoration of skilled forelimb use by ectopic dopaminergic grafts was most successful in "indifferent" animals without a clear lateralization in this specific task, followed by animals that received dopaminergic grafts into the "dominant" hemisphere. Finally, only a very modest improvement was seen in "non-dominant" hemisphere-grafted animals.

In previous studies, dopaminergic grafts largely failed to improve the complex sensorimotor behavior involved in skilled forelimb movements, whereas more simple motor deficits seen in tests of rotation, stepping, and contralateral neglect have demonstrated good or even complete recovery in transplanted animals (for more extensive discussion, see Dunnett et al., 1987; Montoya et al., 1990, 1991; Abrous et al., 1993a,b; Dunnett and Björklund, 1994b; Barker and Dunnett, 1999). A number of hypotheses have been developed to explain the inability of nigral grafts to restore skilled forelimb use in rat models of Parkinson's disease, including graft location, ectopic placement, graft maturation and differentiation, and mechanisms involved in learning and training on how to use the transplant (Björklund et al., 1987, 1994; Barker and Dunnett, 1999; Freed, 2000). However, using a more extensive transplantation approach by which 18-20 dopaminergic micrografts were distributed over the entire head of the caudateputamen, restoring striatal dopaminergic innervation up to 60$90 \%$ of normal values, first evidence for a significant, although incomplete restoration of skilled forelimb use mediated via dopaminergic transplants was observed in some studies (Nikkhah et al., 1993; Winkler et al., 1999). In the first study by Nikkhah et al. (1993), it was noted that the magnitude of improvement in skilled forelimb use differed markedly among the grafted rats. Although good or even normal performance levels were reached in $\sim 60 \%$ of the grafted animals, the remaining $40 \%$ of the transplanted animals performed at a level within the range of lesion-only controls. This difference could not be attributed to graft size, number of surviving TH-positive neurons, or the extent of reinnervation (Nikkhah et al., 1993; Winkler et al., 1999).

In the present study, hemispheric dominance of paw preference could be identified as a critical host-derived factor that governs the development and extent of functional restoration of complex sensorimotor movements involved in skilled forelimb use. Animals without a clear paw preference in forelimb use (indiff $\mathrm{H}-\mathrm{Tx}$ ) seemed to be more amenable to graft-induced functional recovery observed in the paw reaching test. However, this functional improvement only became apparent during the second test 16 weeks after the transplantation procedure. In contrast, tests of rotational asymmetry, stepping, and postural balance all demonstrated good or complete recovery 5 weeks posttransplantation, a time point, at which graft maturation and functional integration is believed to be established in this model (Abrous et al., 1988; Nikkhah et al., 1994a). This also indicates that animals with nigral dopaminergic grafts may need a more extensive training period to learn how to use their transplants in more complex tasks and behaviors, such as skilled forelimb use. In the case of an asymmetrical preference of skilled forelimb use, as seen in the dom H-Tx and non-dom H-Tx groups, functional recovery in paw reaching is delayed and can only be demonstrated by using longer testing periods, e.g., 15-30 min instead of 5 min per test session. The hypothesis that training and learning may be fostered by more extensive testing sessions is further supported by the fact that a significant recovery in skilled forelimb use was only observed in the forced choice test in which the animal has to concentrate on using only the affected forelimb while the other normal forelimb cannot be used because of the design of the staircase apparatus (Montoya et al., 1991). Similar observations on learning how to use a graft have been made with intrastriatal striatal grafts in animal models of Huntington's disease (Mayer et al., 1992; Brastedt et al., 1999); however, in this experimental paradigm, the homotopic striatal graft placement can lead to a reestablishment of the physiological cortico-striatal-pallidal connections (Wictorin, 1992).

The mechanisms underlying the interaction between hemispheric dominance, extensive testing, and dopaminergic graftinduced recovery in skilled forelimb use is likely to be more complex.

There are a number of studies in both mice (Cabib et al., 1995; Biddle and Eales, 1996, 1999; Nielsen et al., 1997) and rats (Schwarting et al., 1987) demonstrating a close correlation between hemispheric dominance (e.g., for paw preference or circling behavior) and dopamine content and metabolism. Schwarting et al. (1987) have shown that dopamine metabolism is increased in the ventral and dorsal striatum, septum, and substantia nigra when paw use is restricted by physical constraints (so called "forced-handedness") as opposed to a nonrestricted paw use (so called "paw preference"). Furthermore, Cabib et al. (1995) have provided evidence for the hypothesis that dopamine and its metabolites are strongly related to both the direction and intensity of behavioral lateralization in paw preference, with a special emphasis on the mesoaccumbens dopamine system. Because of the incomplete anatomical reconstruction achieved by ectopic intrastriatal nigral grafts used in the present study, the underlying mechanisms involved in hemispheric dominance and paw preference and performance may not completely be normal- 
ized, and this may be one explanation why animals without a dominant lateralization of skilled forelimb reaching did show the most substantial recovery of graft-induced function, followed by animals grafted in the dominant hemisphere and, last, in the non-dominant hemisphere. For a further fine-tuning of the functional balance between both hemispheres, a more complete reconstruction of the entire mesostriatal dopamine projection system may be necessary, as has been attempted in neonatal hosts (Wictorin, 1992; Nikkhah et al., 1995a,b) or using the bridge graft technology (Wictorin, 1992; Brecknell et al., 1996; Wilby et al., 1999) (see also Björklund et al., 1994; Winkler et al., 2000).

Because of similarities in forelimb movements observed between humans and rats (Whishaw et al., 1986, 1992) and the analogies between the rat model of PD and the clinical picture of PD, these findings may also have significant implications for current clinical neural transplantation strategies aiming at restoring behavioral symptoms in patients suffering from Parkinson's disease (Freeman and Widner, 1998; Björklund and Lindvall, 2000). It would indicate that not only graft-derived but also host-derived factors determine the final therapeutic outcome, i.e., possibly a reverse relationship between the degree of hemispheric dominance for individual complex sensorimotor symptoms in parkinsonian patients and the restorative capacity of dopaminergic grafts to ameliorate those deficits. Second, further critical variables such as learning and extensive and specific testing during the rehabilitation phase may be important host-derived factors to consider to fully examine and demonstrate the functional integration and therapeutic potential of nigral dopaminergic grafts in Parkinson's disease.

In conclusion, the results of the present study demonstrate that hemispheric dominance in paw preference and extensive testing regimes are critical factors in determining the restorative plasticity of intrastriatally placed DA grafts. In this aspect, the rat model of PD has proven to be a very useful tool to investigate important parameters that can lead to a better understanding of the functional anatomical architecture of the dopaminergic nigrostriatal system and, thereby, promote the development of novel and powerful concepts designed to optimize the anatomical repair and functional reconstruction on the basis of neural transplantation strategies.

\section{REFERENCES}

Abercrombie M (1946) Estimation of nuclear population from microtome sections. Anat Rec 94:239-247.

Abrous DN, Torres EM, Dunnett SB (1993a) Dopaminergic grafts implanted into the neonatal or adult striatum: comparative effects on rotation and paw reaching deficits induced by subsequent unilateral nigrostriatal lesions in adulthood. Neuroscience 54:657-668.

Abrous DN, Shaltot A, Torres EM, Dunnett SB (1993b) Dopamine-rich grafts in the neostriatum and/or nucleus accumbens: effects on druginduced behaviours and skilled paw-reaching. Neuroscience 53:187-197.

Abrous N, Guy J, Vigny A, Calas A, Le Moal M, Herman JP (1988) Development of intracerebral dopaminergic grafts: a combined immunohistochemical and autoradiographic study of its time course and environmental influences. J Comp Neurol 273:26-41.

Annett LE (1994) Functional studies of neural grafts in parkinsonian primates. In: Functional neural transplantation (Dunnett SB, Björklund A, eds), pp 71-102. New York: Raven.

Annett LE, Martel FL, Rogers DC, Ridley RM, Baker HF, Dunnett SB (1994) Behavioral assessment of the effects of embryonic nigral grafts in marmosets with unilateral 6-OHDA lesions of the nigrostriatal pathway. Exp Neurol 125:228-246.

Barker RA, Dunnett SB (1999) Neural repair, transplantation and rehabilitation. East Sussex, UK: Psychology Press Ltd.

Biddle FG, Eales BA (1996) The degree of lateralization of paw usage (handedness) in the mouse is defined by three major phenotypes. Behav Genet 26:391-406.

Biddle FG, Eales BA (1999) Mouse genetic model for left-right hand usage: context, direction, norms of reaction, and memory. Genome 42:1150-1166.

Björklund A (1992) Dopaminergic transplants in experimental parkinsonism: cellular mechanisms of graft-induced functional recovery. Curr Opin Neurobiol 2:683-689.

Björklund A, Lindvall O (2000) Cell replacement therapies for central nervous system disorders. Nat Neurosci 3:537-544.

Björklund A, Stenevi U (1979) Reconstruction of the nigrostriatal dopamine pathway by intracerebral nigral transplants. Brain Res 177:555-560.

Björklund A, Stenevi U, Dunnett SB, Iversen SD (1981) Functional reactivation of the deafferented neostriatum by nigral transplants. Nature 289:497-499.

Björklund A, Stenevi U, Schmidt RH, Dunnett SB, Gage FH (1983) Intracerebral grafting of neuronal cell suspensions. I. Introduction and general methods of preparation. Acta Physiol Scand [Suppl] 522:1-7.

Björklund A, Lindvall O, Isacson O, Brundin P, Wictorin K, Strecker RE Clarke DJ, Dunnett SB (1987) Mechanisms of action of intracerebral neural implants. Trends Neurosci 10:509-516.

Björklund A, Dunnett SB, Nikkhah G (1994) Nigral transplants in the rat Parkinson model. Functional limitations and strategies to enhance nigrostriatal reconstruction. In: Functional neural transplantation (Dunnett SB, Björklund A, eds), pp 47-68. New York: Raven.

Brastedt PJ, Watts C, Robbins TW, Dunnett SB (1999) Associative plasticity in striatal transplants. Proc Natl Acad Sci USA 96:10524-10529.

Brecknell JE, Du JS, Muir E, Fidler PS, Hlavin ML, Dunnett SB, Fawcett JW (1996) Bridge grafts of fibroblast growth factor-4-secreting schwannoma cells promote functional axonal regeneration in the nigrostriatal pathway of the adult rat. Neuroscience 74:775-784.

Brundin P, Duan W-M, Sauer H (1994) Functional effects of mesencephalic dopamine neurons and adrenal chromaffin cells grafted to the rodent striatum. In: Functional neural transplantation (Dunnett SB, Björklund A, eds), pp 9-46. New York: Raven.

Cabib S, D'Amato FR, Neveu PJ, Deleplanque B, Le Moal M, PuglisiAllegra S (1995) Paw preference and brain dopamine asymmetries. Neuroscience 64:427-432.

Dobrossy MD, Le Moal M, Montaron MF, Abrous N (2000) Influence of environment on the efficacy of intrastriatal dopaminergic grafts. Exp Neurol 165:172-183.

Dunnett SB, Björklund A (1994a) Mechanisms of function of neural grafts in the injured brain. In: Functional neural transplantation (Dunnett SB, Björklund A, eds), pp 531-567. New York: Raven.

Dunnett SB, Björklund A (1994b) Functional neural transplantation. New York: Raven.

Dunnett SB, Björklund A (1999) Prospects for new restorative and neuroprotective treatments in Parkinson's disease. Nature 399:32-39.

Dunnett SB, Whishaw IQ, Rogers DC, Jones GH (1987) Dopamine-rich grafts ameliorate whole body motor asymmetry and sensory neglect but not independent limb use in rats with 6-hydroxydopamine lesions. Brain Res 415:63-78.

Freed WJ (2000) Neural transplantation: an introduction. Cambridge, MA: MIT.

Freeman T, Widner H (1998) Cell transplantation for neurological disorders. Totowa, NJ: Humana.

Glick SD, Ross DA (1981) Right-sided population bias and lateralization of activity in normal rats. Brain Res 205:222-225.

Gundersen, Bendtsen TF, Korbo L, Marcussen N, Moller A, Nielsen K, Nyengaard JR, Pakkenberg B, Sorensen SB, Vesterby A, West MJ (1988) Some new, simple, and efficient stereological methods and their use in pathological research and diagnosis. APM IS 96:379-394.

Herman J-P, Abrous N (1994) Dopaminergic neural grafts after fifteen years: results and perspectives. Prog Neurobiol 44:1-35.

Mandel RJ, Brundin P, Björklund A (1990) The importance of graft placement and task complexity for transplant-induced recovery of simple and complex sensorimotor deficits in dopamine denervated rats. Eur J Neurosci 2:888-894.

Mayer E, Brown VJ, Dunnett SB, Robbins TW (1992) Striatal graftassociated recovery of a lesion-induced performance deficit in the rat requires learning to use the transplant. Eur J Neurosci 4:119-126.

Mayhew TM (1992) A review of recent advances in stereology for quantifying neural structure. J Neurocytol 21:313-328.

Miklyaeva EI, Ioffe ME, Kulikov MA (1991) Innate versus learned factors determining limb preference in the rat. Behav Brain Res 46:103-115.

Montoya CP, Astell S, Dunnett SB (1990) Effects of nigral and striatal grafts on skilled forelimb use in the rat. Prog Brain Res 82:459-466.

Montoya CP, Campbell HL, Pemberton KD, Dunnett SB (1991) The 'staircase test': a measure of independent forelimb reaching and grasping abilities in rats. J Neurosci Methods 36:219-228

Nielsen DM, Visker KE, Cunningham MJ, Keller Jr RW, Glick SD, Carlson JN (1997) Paw preference, rotation, and dopamine function in Collins HI and LO mouse strains. Physiol Behav 61:525-535.

Nikkhah G, Duan W-M, Knappe U, Jödicke A, Björklund A (1993) Restoration of complex sensorimotor behavior and skilled forelimb use 
by a modified nigral cell suspension transplantation approach in the rat Parkinson model. Neuroscience 56:33-43.

Nikkhah G, Olsson M, Eberhard J, Bentlage C, Cunningham MG, Björklund A (1994a) A microtransplantation approach for cell suspension grafting in the rat Parkinson model. A detailed account of the methodology. Neuroscience 63:57-72.

Nikkhah G, Cunningham MG, Jödicke A, Knappe U, Björklund A (1994b) Improved graft survival and striatal reinnervation by microtransplantation of fetal nigral cell suspensions in the rat Parkinson model. Brain Res 633:133-143.

Nikkhah G, Cunningham MG, Cenci MA, McKay R, Björklund A (1995a) Dopaminergic microtransplants into the substantia nigra of neonatal rats with bilateral 6-OHDA lesions. I. Evidence for anatomical reconstruction of the nigrostriatal pathway. $J$ Neurosci 15:3548-3561.

Nikkhah G, Cunningham MG, McKay R, Björklund A (1995b) Dopaminergic microtransplants into the substantia nigra of neonatal rats with bilateral 6-OHDA lesions. II. Transplant-induced behavioral recovery. J Neurosci 15:3562-3570.

Nikkhah G, Rosenthal C, Hedrich HJ, Samii M (1998) Differences in acquisition and full performance in skilled forelimb use as measured by the 'staircase test' in five rat strains. Behav Brain Res 92:85-95.

Nikkhah G, Winkler C, Rödter A, Samii M (2000) Microtransplantation of nigral dopamine neurons: a "step-by-step" recipe. In: Neural transplantation methods (Dunnett SB, Baker G, eds), pp 207-231. Totowa, NJ: Humana.

Olsson M, Nikkhah G, Bentlage C, Björklund A (1995) Forelimb akinesia in the rat Parkinson model: differential effects of dopamine agonists and nigral transplants as assessed by a new stepping test. J Neurosci 15:3863-3875.

Pearlson GD, Kubos KL, Robinson RG (1984) Effect of anteriorposterior lesion location on the asymmetrical behavioral and biochemical response to cortical suction ablations in the rat. Brain Res 293:241-250.

Perlow MI, Freed WJ, Hoffer BJ, Seiger A, Olson L, Wyatt RJ (1979) Brain grafts reduce motor abnormalities produced by destruction of the nigrostriatal dopamine system. Science 204:643-647.
Schallert T, Hall S (1988) Disengage sensorimotor deficit following apparent recovery from unilateral dopamine depletion. Behav Brain Res 30:15-24.

Schallert T, Norton D, Jones TA (1992) A clinically relevant unilateral rat model of Parkinsonian akinesia. J Neural Transplant Plast 3:332-333.

Schwarting R, Nagel JA, Huston JP (1987) Asymmetries of brain dopamine metabolism related to conditioned paw usage in the rat. Brain Res 417:75-84.

Ungerstedt U, Arbuthnott G (1970) Quantitative recording of rotational behavior in rats after 6-hydroxy-dopamine lesions of the nigrostriatal dopamine system. Brain Res 24:485-493.

Whishaw IQ, O'Connor WT, Dunnett SB (1986) The contributions of motor cortex, nigrostriatal dopamine and caudate-putamen to skilled forelimb use in the rat. Brain 109:805-843.

Whishaw IQ, Pellis SM, Gorny BP (1992) Skilled reaching in rats and humans: evidence for parallel development or homology. Behav Brain Res 47:59-70.

Wictorin K (1992) Anatomy and connectivity of intrastriatal striatal transplants. Prog Neurobiol 38:611-639.

Wilby MJ, Sinclair SR, Muir EM, Zietlow R, Adcock KH, Horellou P, Rogers JH, Dunnett SB, Fawcett J (1999) A glial cell line-derived neurotrophic factor-secreting clone of the Schwann cell line SCTM41 enhances survival and fiber outgrowth from embryonic nigral neurons grafted to the striatum and to the lesioned substantia nigra. J Neurosci 19:2301-2312.

Winkler C, Sauer H, Lee CS, Björklund A (1996) Short-term GDNF treatment provides long-term rescue of lesioned nigral dopamine neurons in a rat model of Parkinson's disease. J Neurosci 16:7206-7215.

Winkler C, Bentlage C, Nikkhah G, Samii M, Björklund A (1999). Intranigral transplants of GABA-rich striatal tissue induce behavioral recovery in the rat Parkinson model and promote the effects obtained by intrastriatal dopaminergic transplants. Exp Neurol 155:165-186.

Winkler C, Kirik D, Björklund A, Dunnett SB (2000) Transplantation in the rat model of Parkinson's disease: ectopic versus homotopic graft placement. In: Functional neural transplantation, (Dunnett SB, Björklund A, eds), pp 233-265. Amsterdam: Elsevier. 